\title{
Identification of Drivers for Modular Production
}

\author{
Thomas Ditlev Brunoe, Jacob Bossen, Kjeld Nielsen \\ Department of Mechanical and Manufacturing Engineering, Aalborg University, Fibiger- \\ straede 16, 9220 Aalborg East, Denmark \\ tdp@m-tech.aau.dk
}

\begin{abstract}
Todays competitive environment in industry creates a need for companies to enhance their ability to introduce new products faster. To increase ramp-up speed reconfigurable manufacturing systems is a promising concept, however to implement this production platforms and modular manufacturing is required. This paper presents an analysis whether and which module drivers from general product development can be applied to the development process of a modular manufacturing system. The result is a compiled list of modular drivers for manufacturing and examples of their use.
\end{abstract}

Keywords: Production platforms, Drivers, Modular Manufacturing

\section{$1 \quad$ Introduction}

It is generally accepted that the dynamics of markets of today call for higher product variety as well as reduced product life cycles compared to just one or two decades ago [4]. This calls for manufacturing companies to become more efficient in introducing new products into the production system and ramping up production. As product lifecycles shorten, it becomes increasingly critical to minimize the investments in establishing and ramping up production, since the time for return of investment in the manufacturing system incurs over a shorter period than previously. For this reason, reuse of parts of or reuse of entire production systems becomes increasingly relevant, as this is one means to reduce cost of investments related to new product introduction in the manufacturing system. One way to achieve this, is by implementing a flexible manufacturing system, a system that consists of generic manufacturing equipment, e.g. CNC mills, which can perform a wide variety of manufacturing tasks, but is relatively inefficient compared to a dedicated manufacturing system, which on the other hand has very little flexibility [4]. Introduced as a new class of production systems, reconfigurable manufacturing systems as introduced by Koren [4] realizes a predefined level of flexibility, but reaches a level of efficiency near that of dedicated manufacturing systems.

A major enabler for implementing reconfigurable manufacturing systems, as well as a major enabler for reusing assets between different dedicated manufacturing systems in a company, or reusing assets from one manufacturing system generation to another is modularity.

adfa, p. 1, 2011.

(C) Springer-Verlag Berlin Heidelberg 2011 
The concept of product platforms is relatively well described in literature, whereas less literature is to be found concerning production platforms. Product platforms are defined differently in literature; however in this paper the definition by Ulrich \& Eppinger: "... the set of assets shared across a set of products. Components and subassemblies are often the most important of these assets" is adopted [11]. Modularity is closely related to platforms although fundamentally a different concept. Modularity of a system implies that the system is divided into a number of well-defined modules which each implement well defined functions separately, and the interfaces to each module are as simple as possible and well defined. Other research describes the relation between modularity and platform architecture: "platform architecture displays a special type of modularity, in which a product or system is split into a set of components with low variety and high reusability, and another set with high variety and low reusability" [1]. This implies that modularity enables a platform architecture, and thus platform architectures will always be modular, while modular architectures may not always apply platform architectures.

The concepts of modularity and platform architecture have been adopted to the production domain, and labelled production platforms [7], [10] and modular manufacturing. The basic concepts of modularity and platform architecture can be altered directly from products to production however; specific methods for designing manufacturing systems introduced as generic product development modularization methods are not directly applicable. The before mentioned modular manufacturing systems are though depended on a structured co-development and co-evolution strategy with supporting design methodologies.

Platform-based Co-development is essentially a research field within the discipline of concurrent engineering and integrated product development, where cross-functional work and information sharing is in focus. The idea is to create and intelligently visualise boundaries for product and production system design in a co-development process, such that variety is only present where it is desirable and acceptable [9]. Early studies of platform-based co-development had inclusions of production aspects in product platform design [8], hence production system assets can be reused

After the definition on product platforms [8], the term Process Platform was coined conceptual wise [3] to underline the production aspect, and later the term was elaborated in another study [15]. Most of the literatures on process platforms focus around generic product and process structures, generic routing, generic planning and generic variety representation. Even though the process platform literature focuses on both product and process structure, the literature lacks support for co-development where a more holistic view is essential.

Research on modular product development states that in order to achieve the optimal product architecture, different criteria for performing the modularization must be considered and prioritized [2], [6], [11]. These criteria are referred to by some as module drivers [6]. Considering different criteria when modularizing a production system or establishing a production platform is also considered of paramount importance, however in the literature reviewed in this research, no systematic exploration of production platform drivers has been identified. 


\subsection{Resarch Question and method}

The objective of this paper is thus to contribute to modular production development and the area of production platform development by identifying module drivers in production. Since module drivers in product development are well described, we will take the approach to evaluate different module drivers from product development and adopt those to a production system context. The research question of this paper is thus: Which module drivers from product development can be translated into drivers for modules in production systems and production platforms?

The research question has been addressed by reviewing literature and identifying literature describing module drivers in general product development. Each of the identified drivers is evaluated to determine whether they make sense in terms of using them in a production system context and a new definition is proposed which directly addresses modules in production systems.

The basis for evaluating and formulating the module drivers is primarily the work of Ulrich and Eppinger [11] and Ericsson \& Erixon [2], as these two frameworks by far present the most elaborate set of module drivers. As described by Wiendahl et al. [13], production system changeability and thereby also modularity is relevant om multiple levels. These levels are defined as 1) station, 2) Cell, 3) System, 4) Segment, 5) Factory, and 6) Network. Modularity in production systems can be relevant at each level, meaning that e.g. a line can be perceived as a module, just as well as a station or an entire factory can be defined as modules. We would even consider lower levels as candidates for modules, e.g. machines, tools or parts of tools, as suggested by Koren [5]. Hence, the examples given below will refer to various levels in production systems to illustrate the diversity of the concept of modularity in manufacturing.

\section{$2 \quad$ Identification of Drivers}

In relation to the method modular function deployment a number of module drivers are proposed [2], [6]. These drivers are outlined below: 1) Module carryover, 2) technology Evolution, 3) Planned product Changes, 4) Different Specification, 5) Variety Styling, 6) Common unit, 7) Process and/or Organization), 8) Separate Testing, 9) Supplier available, 10) Service and Maintenance, 11) Upgrading), and 12) Recycling.U1rich \& Eppinger [11] identified a number of factors which are essential to consider when clustering product functions in chunks, which is their terms for defining the modularity. These factors translate directly into module drivers using the terminology of Ericsson and Erixon [2]. These factors are outlined in the following and are to some extent similar, however this will be addressed in the following and a complete list of module drivers will be presented: 1) Geometric Integration and Precision, 2) Function Sharing, 3) Capabilities of Vendors, 4) Similarity of Design or Production Technology, 5) Localization of Change, 6) Accommodating Variety, 7) Enabling Standardization, and 8) Portability of Interfaces.

In the following, the identified primary module drivers will be described in more detail. To combine the different module drivers identified above, five categories are 
used: 1) Localization of changes in product, 2) Variety and standardization, 3) Production 4) Service and Recycling 5) Product development.

\subsection{Product Development}

The module driver geometric integration and precision is relevant in products where certain components need to be very carefully aligned for the product to function. Examples of this include cameras, where the optics and sensors need to be very carefully positioned to function correctly. By integrating such components in the same module, there is a lower risk of quality issues in the finished product, since assembly precision will not be an issue in the final assembly of the product. We consider this driver relevant to production modularity, since e.g. assembly operations in manufacturing need very precise alignment of parts and equipment positioning these parts, e.g. manipulators must be very precisely aligned to function properly, and this would likely promote these different functions to be incorporated in the same module of the manufacturing system. Another module driver is function sharing. This refers to cases where two functions in a product can share some kind of sub-function. This module driver is widely applied in design of electronics, where certain functions may be implemented on the same circuit board to share functions like power supply, a micro-processor or cooling. By sharing a support function, the cost of developing producing that support function is saved and the size of the final product may be reduced. In a manufacturing context an example of function sharing within a module could be a robot welding cell, here considered one module, where two fixtures share one welding robot. In this case, because the fixtures "share" the welding robot, a higher utilisation of the robot can be achieved as one fixture can be loaded while the robot welds on the other fixture. Michaelis provides an industrial example of this [9].

In some product types, the portability of interfaces is an essential module driver. Components which have interfaces which are not easily portable will usually be beneficial to implement in one module. The portability of an interface is defined by how easy it is to place two components with a common interface apart from each other. Some specific types of interfaces are more easily portable than others. For example electrical interfaces are usually more portable than mechanical or force transmitting interfaces. This driver is also considered relevant in production systems, since interfaces between different manufacturing equipment also have different portability. Consider painting and drying operations in a production. These two operations must be located next to each other to minimize transport, as transport of wet paint would damage the paint, due to dust and impurities. Other interfaces are more portable, e.g. data interfaces between a control module and a mechanical machine module.

\subsection{Localization of changes}

Module carryover refers to cases where some function in the product is not expected to change for a number of product generations. In these cases it is usually beneficial to cluster this function in a module since this module can be 'carried over' more or less 
unchanged to later generations of the product [2]. This implies that the cost of developing that module type will not occur in subsequent development projects using the module, leading to reduced development cost.

Technology evolution refers to parts of a product where a change can be expected which is caused by demand for new technology, the presently used technology becomes obsolete or opportunities regarding e.g. new materials are introduced [2]. In manufacturing, this could be e.g. a shift from arc welding to laser welding that requires new equipment, and by isolating the welding equipment in one module, the propagation of change can be contained. Planned product changes are similar to technology evolution, however, what drives these changes in the product is not technology but rather product changes, which are part of a product plan e.g. to provide new functionality to the customer. Although in some cases, this would imply using new technology as described above this is not always the case and the motivation for making the change is fundamentally different. In manufacturing systems, this would translate into changes in production capacity or capability, meaning that some production modules may be defined, e.g. an expensive bottleneck operation, which needs to be upgraded when increased production capacity is required.

The benefits of implementing elements, which are expected to change, either due to technology evolution or due to planned changes, are very similar. By clustering these elements in one or a few modules, the change will be focused in that particular module and thus the rest of the production system may remain unchanged. Assuming that making changes in fewer modules leads to lower cost, clustering elements expected to change will reduce development costs for changing the production system to meet future requirements.

\subsection{Variety and standardization}

Although contrasting concepts, in relation to modular products, variety and standardization are closely related. It is widely acknowledged that modular product design is an efficient means to achieve a high product variety at low costs. The reason for this is that a product family designed with interfaces supporting exchange of modules may present the customer with a very large variety by combining even a small number of modules.

Ericsson and Erixon [2] have classified the variety into product specification and styling variety, where product specification refers to the actual functionality of the product, whereas styling only refers to the appearance. Since production equipment is seldom specified in terms of aesthetics, we consider the styling driver irrelevant,

The module driver common unit or enabling standardization is the idea of identifying a function which is required in a number of different products in a product portfolio, developing a module implementing this function and utilize it in all of these products. By doing this, development effort is saved, since the module needs only to be designed once, and economies of scale can be achieved in production with lower unit costs as a result [2], [12].

Considering production systems with multiple similar parallel operations, as for example injection moulding of different plastic parts, it may be beneficial to define a 
"standard module" consisting of a moulding machine and material feeder and a variety carrying module consisting of moulding forms. In this case, any machine (standard module or common unit) can manufacture any part if combined with the corresponding mould, leading to increased flexibility in planning and increased utilization, compared to dedicated machines. Hence the module drivers for "common unit" and "different specification" are highly relevant in a production system context.

\subsection{Production}

Establishing modules based on similarity in production technology and testing can greatly reduce the manufacturing costs of a module. Consider a modular product requiring three different production technologies, which is available in three different manufacturing lines. If each module requires different manufacturing technologies, the module would have to be moved between manufacturing lines, increasing the lead time and manufacturing costs. Although similarity in production technology and testing may seem relevant when defining production system modules, this is already covered by the "common unit driver" as the production drivers described in this section refer to the production of the focal system, which in this case is the manufacturing system itself. Hence these drivers would refer to the production of e.g. the machines used in the production, which we consider less relevant when designing a production system. We therefore do not include these drivers.

Vendor capabilities can also be a driver for establishing a module. If the development and manufacturing of certain functions in a product is outsourced to external partners, having those functions implemented in a single or few modules will allow easier specification of interfaces and reduced complexity in the assembly process. Furthermore, certain functions may be available from specialized suppliers as standard modules, which would remove development cost and reduce purchase costs as well. This driver is also considered relevant for manufacturing systems as certain manufacturing operations need highly customized equipment delivered from very specialized suppliers, and focusing this functionality in fewer modules reduces complexity and reduces the number of modules developed by external vendors.

\subsection{Service and Recycling}

During a products life cycle after it has been sold, a number of factors may also be important to consider when defining modules. For service and maintenance purposes, it may be beneficial to simply replace a defective module rather than repairing it while installed in the product. Related to manufacturing systems this drivers is also considered relevant, as being able to interchange a module with a replacement module once it fails, is preferable compared to stopping the whole production system while repairing the failed component. Examples of this could be replaceable cutting tools for mills or lathes, which can easily be replaced instead of sharpening them while in the machine. Modules may also be defined to allow easy upgrading of a product. Modules allowing upgrades are also relevant in manufacturing systems, however these are already covered 
by the driver "planned changes", and is therefore omitted from the final set of module drivers.

The extent, to which a product can be recycled, depends to a large degree on how easy it is to disassemble and separate material into fractions. Hence designing modules that support this would increase the recyclability of the product as a whole. While this driver is very important when designing products, because they are manufactured in large numbers, this is less relevant for designing production systems, since usually only one or a few production systems are implemented. Furthermore, the expected lifespan of a production system is much longer than a typical consumer product and hence the recyclability is less important to consider when designing the system, although relevant for the manufacturers of the equipment (robot companies for example), as they manufacture large numbers of each machine.

\section{Summary of Results}

In the sections above, module drivers from product development have been evaluated, using the criteria whether they are relevant when designing a modular production system on various levels. Each driver identified as relevant is presented in the table below.

As presented above, many of the module drivers originally described for product development are relevant for production systems as well. Many of the examples presented above for each module driver are even well known design principles for manufacturing systems currently applied widely in industry. However, by identifying the different drivers and explicitly evaluating how they should be prioritised can be a means towards making decisions about modularising production systems, where it is more likely to focus on the module drivers, which are most beneficial in each specific case, thus leading to a better production system.

\section{Conclusion}

The drivers for modules in production systems presented in this paper are basically expected benefits of defining module in a certain way, and many of these drivers are contradicting, e.g. you cannot accommodate standardization and variety in the same

Table 1. Overview of module Drivers for modules in a manufacturing system

\begin{tabular}{|cc|}
\hline \multirow{3}{*}{ System development } & Geometric integration and precision \\
\cline { 2 - 2 } & Function Sharing \\
\cline { 2 - 2 } Localization of Changes & Portability of Interfaces \\
\cline { 2 - 2 } & Module Carryover \\
\cline { 2 - 2 } & Technology Evolution \\
\hline \multirow{2}{*}{ Variety and Standardization } & Common Unit \\
& Different Specification \\
\hline Production of Manufacturing Equipment & Vendor Capabilities \\
\hline Service and Recycling & Service and Maintenance \\
\hline
\end{tabular}


module. Hence, these drivers should be used in relation to a development method for designing a modular production system. Some methods exist for this and it is the authors' expectation that also product modularization methods, such as Modular Function Deployment of Design Structure Matrix, can be adapted to modular production system development. However, the validation of this will be topic of future research.

The drivers presented in this paper have been identified from literature concerning product development. Hence, we cannot conclude that no other drivers exist for development of manufacturing systems, which do not have a product development equivalent. Hence, the drivers that are unique to production system development are not identified in this paper. This will be a topic of future research.

The results of this research, the set of and description of the drivers is expected to be relevant to academics for future research in modular manufacturing as well as practitioners designing new modular production systems and production platforms.

\section{References}

1. Baldwin, C. Y., \& Woodard, C. J.: The architecture of platforms: a unified view. In: Gawer, A. (ed.) Platforms, Markets and Innovation, pp. 19. EE Publishing, Cheltenham, UK (2009)

2. Ericsson, A., \& Erixon, G.: Controlling design variants: Modular product platforms. ASME Press (1999)

3. Jiao, J., Zhang, L., Pokharel, S.: Process Platform Planning for Mass Customisation Production. International Journal of Mass Customisation, 1 (2006) 237-259

4. Koren, Y.: The global manufacturing revolution: Product-process-business integration and reconfigurable systems. John Wiley \& Sons (2010)

5. Koren, Y.: The global manufacturing revolution: Product-process-business integration and reconfigurable systems. John Wiley \& Sons (2010)

6. Lange, M. W., \& Imsdahl, A.: Modular Function Deployment: Using Module Drivers to Impart Strategies to a Product Architecture. In: Anonymous Advances in Product Family and Product Platform Design, pp. 91-118. Springer (2014)

7. Levandowski, C., Michaelis, M. T., Johannesson, H.: Set-Based Development using an Integrated Product and Manufacturing System Platform. Concurrent Engineering, 22 (2014) 234-252

8. Meyer, M. H., \& Lehnerd, A. P.: The power of product platforms: Building value and cost leadership. New York: Free Press (1997)

9. Michaelis, M. T.: Co-development of products and manufacturing systems using integrated platform models. Chalmers University of Technology (2013)

10. Michaelis, M. T.: Co-Development of Products and Manufacturing Systems-Applying a Configurable Platform Approach. (2011)

11. Ulrich, K. T., \& Eppinger, S. D.: Product design and development, Vol. 2. McGrawHill/Irwin New York (2011)

12. Ulrich, K., \& Eppinger, S. D.: Product design and development. McGraw-Hill (2003)

13. Wiendahl, H., ElMaraghy, H. A., Nyhuis, P. et al.: Changeable Manufacturing-Classification, Design and Operation. CIRP Annals-Manufacturing Technology, 56 (2007) 783809

14. Zhang, L. L.: Process platform-based production configuration for mass customization., (2007) 